\title{
Anther culture performance in selected high yielding indica (of Sri Lanka) and japonica rice varieties and their inter sub-specific hybrids
}

\author{
H.M.I. Herath ${ }^{1 *}$ and D.C. Bandara ${ }^{2}$ \\ ${ }^{I}$ Postgraduate Institute of Agriculture, University of Peradeniya, Peradeniya. \\ ${ }^{2}$ Department of Agricultural Biology, Faculty of Agriculture, University of Peradeniya, Peradeniya.
}

\begin{abstract}
The ability to improve anther culture response of some high yielding commercially grown indica rice varieties by crossing them with a high anther culture responding japonica rice variety was studied. Performance of japonica rice variety $\mathrm{Hu}$ lo tao, indica rice varieties Bg 90-2, Bg 379-2, Bg 94-1 and their F1 hybrids and F2 plants were investigated for callus induction and plant regeneration. Panicles were stored at $8{ }^{\circ} \mathrm{C}$ for 14 days as the cold pre-treatment and anthers were cultured in Chu's N6 medium with 5\% (w/v) sucrose, $2.0 \mathrm{mg} \mathrm{L}^{-1} 2,4$ dichloro phenoxy acetic acid $(2,4 \mathrm{D})$ and $0.5 \mathrm{mg} \mathrm{L}^{-1} \mathrm{Kinetin}_{\text {. The }}$ cultures were kept in the dark at $28 \pm 2{ }^{\circ} \mathrm{C}$ for callus induction. Induced calli of 1-2 $\mathrm{mm}$ diameter were transferred to half strength Murashige and Skoog (MS) medium supplemented with $2.0 \mathrm{mg} \mathrm{L}^{-1}$ Kinetin and $0.5 \mathrm{mg} \mathrm{L}^{-1}$ naphthalene acetic acid (NAA). Of the parental varieties, Hu lo tao is more responsive to anther culture. F2 plants, compared to F1 hybrids and parental rice varieties, had greater callus induction frequencies and plant regeneration. Calli from F1 hybrids and F2 plants of $\mathrm{Bg} 90-2 \times$ $\mathrm{Hu}$ lo tao showed the highest green plant regeneration $(63.3 \%$ and $69.7 \%$ respectively). The regeneration of F1hybrids and F2 plants were higher than in the parents. According to the results, it is possible to improve the anther culture performance of F1 hybrids and F2 plants by crossing high yielding indica rice varieties with high anther culture responding japonica varieties.
\end{abstract}

Keywords: Anther culture, callus induction, indica, japonica, plant regeneration, rice.

\section{INTRODUCTION}

Rice anther culture is an effective and time saving technology for obtaining homozygous lines in varietal improvement (Chu et al., 2002). In the recent past, anther culture in rice has been improved substantially (Roy \& Mandal, 2005). Anther culture has become a powerful tool for the rapid production of haploid and inbred lines used for obtaining hybrid cultivars. Use of doubled haploids in breeding programmes can thus greatly reduce the time required for development of improved cultivars. However, the usefulness of this approach is limited because some genotypes respond poorly to anther culture (Sopory \& Munshi, 1996; Reed, 2002). Practical application of androgenic haploids in the improvement of rice is still limited by the low number of doubled haploids recovered, particularly in indica type (Senadheera et al., 2002). The low percentage of callus induction and green plant regeneration has limited the application of anther culture techniques in indica rice breeding programmes (He et al., 2006). A genetic approach to improve plant regeneration would be the selection for accumulation of favourable alleles for regenarability into a single presumably superior genotype. Plant regeneration from cultured tissues has been shown to be under genetic control in a number of species (Bregitzer \& Campbell, 2002). By understanding the inheritance patterns of anther culture response, breeders can overcome genotype differences by crossing highly responsive to non responsive genotypes and can, to some extent, predict the level of response of the hybrids (Hou et al., 1994). Significant genotypic differences have been observed for callus induction and plant regeneration among the different hybrids of indica and japonica (Narasimman \& Rangasamy, 1993).

Anther culture has been identified as the most appropriate technique at present to improve rice cultivars in Sri Lanka (Mendis et al., 1993). High yielding commercially grown rice varieties with desirable characteristics in Sri Lanka are of indica type, which show poor response to anther culture. Transfer of anther 
culture response traits from japonica to indica rice varieties by crossing can be beneficial to improve indica rice varieties. Only few studies have been conducted on anther culture of F1 hybrids from crosses of cultivated indica and japonica rice varieties (Mendis et al.,1993; Herath et al., 2007). It is important to investigate whether good combinations with high callus induction frequency and green plant regeneration could be selected by hybridization of suitable parents. The aim of this work was to study the ability to improve anther culture response in the $\mathrm{F} 1$ hybrids and $\mathrm{F} 2$ plants by crossing some high yielding, commercially-grown indica rice varieties with a japonica rice variety, which is highly responsive to anther culture.

\section{METHODS AND MATERIALS}

Plant material: Seeds of parental japonica (Hu lo tao) and indica (Bg 90-2, Bg 379-2, Bg 94-1) varieties were obtained from the Plant Genetic Resources Centre (PGRC), Gannoruwa (Table 1). They were grown in a greenhouse and crosses of indica $\times$ japonica (Table 2) were conducted to obtain F1 hybrids. The F1 hybrids were self-fertilized to produce F2 progeny. Seeds of parents, F1 hybrids and F2 plants were sown in pots and the plants were grown in a greenhouse following the standard agronomic practices.

Table 1: Genotype, variety and characteristics of parental rice varieties

\begin{tabular}{|c|c|}
\hline $\begin{array}{l}\text { Genotype } \\
\text { and variety }\end{array}$ & Characteristics \\
\hline Hu lo tao & japonica variety, highly responsive to anther culture \\
\hline BG 90-2 & indica variety, high yielding \\
\hline BG 379-2 & indica variety, high yielding, resistant to Brown \\
\hline BG 94-1 & $\begin{array}{l}\text { Plant Hopper and bacterial blight } \\
\text { indica variety, high yielding }\end{array}$ \\
\hline
\end{tabular}

Anther pre-treatment: First two to three panicles from plants of each genotype were harvested between 9.00 to 10.00 a.m. on sunny days. Panicles were harvested when the distance between collar of flag leaf and penultimate leaf was about 5-7 cm (Croughan, 1998) and the anthers were at uninucleate stage (by microscopic observation), wrapped in aluminium foil with a moistened cotton plug at the base and sealed in polypropylene bags separately. Panicles were stored at $8{ }^{\circ} \mathrm{C}$ for $14 \mathrm{~d}$ (Herath et al., 2009) as the cold pre-treatment. The intact panicles were rinsed with $70 \%(\mathrm{v} / \mathrm{v})$ ethanol for $20 \mathrm{~s}$. Spikelets were then removed and surface sterilized with $30 \%(\mathrm{v} / \mathrm{v})$ commercial bleach solution $\left(\mathrm{Chlorox}^{\circledR}\right)$ for $20 \mathrm{~min}$ and rinsed thoroughly with sterilized distilled water.
Anther culture: Spikelets were cut at the base and the anthers were gently squeezed out with a needle. Hundred anthers were inoculated in $100<15 \mathrm{~mm}$ petri dishes with agar solidified N6 medium (Chu, 1978) supplemented with $2.0 \mathrm{mg} \mathrm{L}^{-1} 2,4$ - dichlorophenoxyacetic acid (2, 4-D), $0.5 \mathrm{mg} \mathrm{L}^{-1}$ Kinetin and 5\% (w/v) sucrose. One petri dish constituted one replicate and an average of 10 replicates were cultured for each genotype. The cultures were placed in the dark at $28 \pm 2{ }^{\circ} \mathrm{C}$ (Chen et al., 1991) for callus induction and examined at weekly intervals for six wks and the percentage of anthers forming calli (callus induction frequency) was recorded after six wks.

Plant regeneration: When the calli were approximately $1-2 \mathrm{~mm}$ in diameter, they were transferred to $100<15 \mathrm{~mm}$ petri dishes containing $25 \mathrm{~mL}$ of half strength Murashige and Skoog (MS) medium (Murashige \& Skoog, 1962) supplemented with $2.0 \mathrm{mg} \mathrm{L}^{-1}$ Kinetin and $0.5 \mathrm{mg} \mathrm{L}^{-1}$ naphthalene acetrc acid (NAA) (Croughan \& Chu, 1991). The cultures were subject to a $16 \mathrm{~h}$ photoperiod $(50 \mu \mathrm{E}$ $\left.\mathrm{m}^{-2} \mathrm{~s}^{-1}\right)$. Observations were made at weekly intervals and the data on percentages of calli regenerating green and/ or albino plants were recorded after 6 wks of incubation. The experiment was repeated thrice and the means were taken for analyses.

The regenerated shoots were transferred to hormonefree, half strength MS medium for rooting. Rooted plants were acclimatized and grown to maturity on paddy soil in a greenhouse under standard agronomic practices. At maturity, spikelet fertility was measured as the ratio of number of grains per panicle to the total number of spikelets per panicle and expressed as a percentage.

Data analyses: Data analyses was done using the Statistical Analyses System (Release 9.1), Analysis of Variance (ANOVA). Mean separation was done by Duncan's multiple range test (DMRT). All experiments were analyzed as a completely randomized design.

\section{RESULTS}

\section{Callus induction}

Callus induction commenced three weeks from culture and the frequency of the anthers forming calli varied between $4.3 \%$ and $78.3 \%$ depending upon the genotype (Figure 1a, 1b, 1c; Table 2). There were strong genotypic effects on callus induction frequency among F1 hybrids, F2 plants and parental rice varieties. Of the parental varieties, $\mathrm{Hu}$ lo tao (japonica) is more responsive to anther culture with $34.3 \%$ callus induction frequency. Out of indica varieties, Bg 90-2 had a significantly higher callus induction frequency than the other two tested 
Table 2: Callus induction frequency and plant regeneration in anther cultures of selected indica, japonica rice varieties, their F1 hybrids and F2 plants. (Significance level $5 \%$ )

\begin{tabular}{lcccc}
\hline Genotype & $\begin{array}{c}\text { Callus induction } \\
\text { frequency (\%) }\end{array}$ & \multicolumn{3}{c}{ Percentage of calli regenerating plants } \\
& & Green (G) & Albino (A) & G/A \\
\hline Parents & $10.5^{\mathrm{g}}$ & $2.2^{\mathrm{g}}$ & $5.1^{\mathrm{f}}$ & 0.43 \\
Bg 90-2 & $6.7^{\mathrm{h}}$ & 0.0 & 0.0 & 0.0 \\
Bg 379-2 & $4.3^{\mathrm{h}}$ & 0.0 & 0.0 & 0.0 \\
Bg 94-1 & $34.3^{\mathrm{e}}$ & $29.3^{\mathrm{c}}$ & $26.3^{\mathrm{c}}$ & 1.11 \\
Hu lo tao & & & & \\
& & & & \\
F1 hybrids & $69.8^{\mathrm{b}}$ & $63.3^{\mathrm{b}}$ & $57.8^{\mathrm{a}}$ & 1.09 \\
Bg 90-2 $\times$ Hu lo tao & $48.2^{\mathrm{d}}$ & $13.8^{\mathrm{e}}$ & $15.1^{\mathrm{d}}$ & 0.91 \\
Bg 379-2 $\times$ Hu lo tao & $23.5^{\mathrm{f}}$ & $7.2^{\mathrm{f}}$ & $11.3^{\mathrm{e}}$ & 0.63 \\
Bg 94-1 $\times$ Hu lo tao & & & & \\
F2 plants & & & & \\
Bg 90-2 $\times$ Hu lo tao & $78.3^{\mathrm{a}}$ & $69.7^{\mathrm{a}}$ & $48.2^{\mathrm{b}}$ & 1.44 \\
Bg 379-2 $\times$ Hu lo tao & $57.6^{\mathrm{c}}$ & $28.4^{\mathrm{c}}$ & $29.7^{\mathrm{c}}$ & 0.95 \\
Bg 94-1 $\times$ Hu lo tao & $34.7^{\mathrm{e}}$ & $20.9^{\mathrm{d}}$ & $23.8^{\mathrm{d}}$ & 0.87 \\
\hline
\end{tabular}

* In each column the means with same superscript letters are not significantly different $(\mathrm{p}<0.05)$
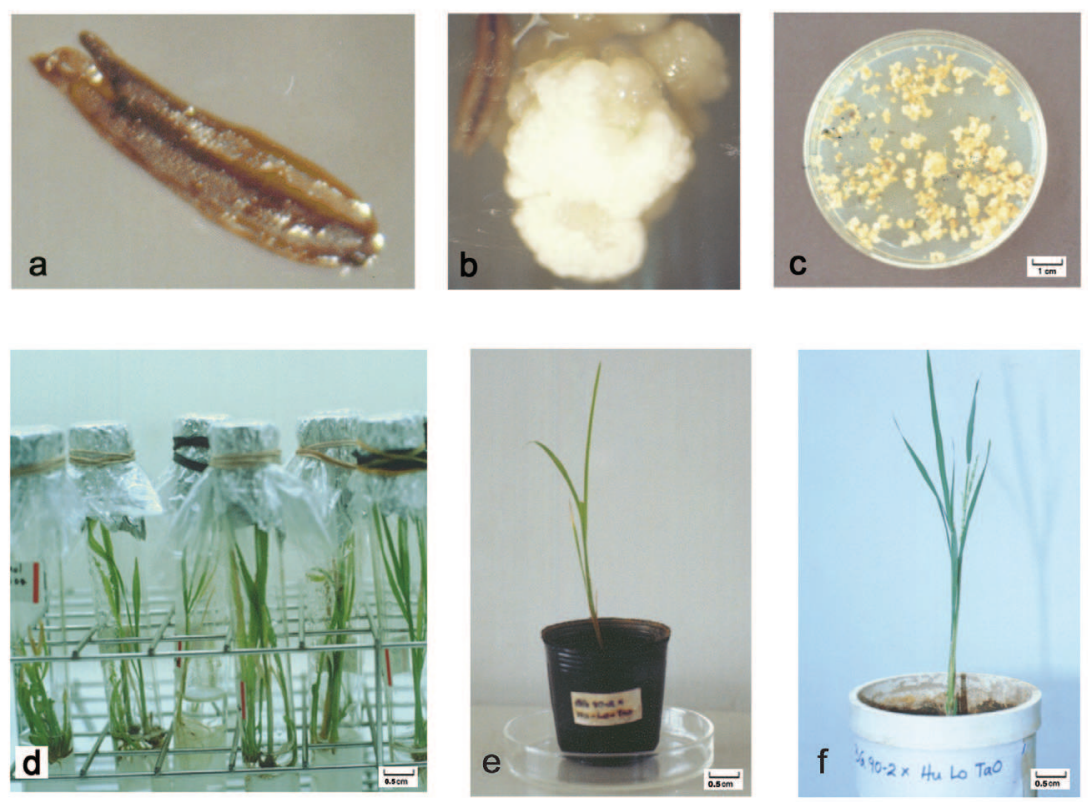

Figure 1: Anther culture response of indica $\times$ japonica crosses

(a) A magnified rice anther after 4 weeks of culture (b) A magnified callus developed after 6 weeks from culture establishment, (c) Callus development after 6 weeks from culture establishment, (d) Green plants regenerated from anther derived calli, (e) Acclimatized anther culture derived rice plantlet and (f) Transplanted rice plantlet of Bg 90-2 $\times$ Hu lo tao F1 hybrid [magnification of (a): $1 \times 100$, (b): $1 \times 40$. Bar in panel (c) $=1.0 \mathrm{~cm}$, bars in panels (d), (e), (f) $=0.5 \mathrm{~cm}]$ 
indica types. The F2 plants, compared to F1 hybrids and parental rice varieties, had greater callus induction frequencies. All crosses except $\mathrm{Bg} 94-1 \times \mathrm{Hu}$ lo tao had significantly higher callus induction frequencies than the japonica parent (Hu lo tao). The highest callus induction frequency in F1 hybrids and F2 plants $(69.8 \%$ and $78.3 \%$ respectively) was observed in $\mathrm{Bg} 90-2 \times \mathrm{Hu}$ lo tao cross.

\section{Plant regeneration}

Frequency of green plant regeneration initiated from calli varied between 2.2 to $69.7 \%$ depending upon the parental genotypes, F1 hybrids and F2 plants (Figure 1d; Table 2). Among the parental rice varieties $\mathrm{Hu}$ lo tao variety was relatively more responsive to green shoot regeneration. Calli from $\mathrm{F} 1$ hybrids and $\mathrm{F} 2$ plants of $\mathrm{Bg}$ 90-2 $\times$ Hu lo tao cross responded better with regard to green plant regeneration when compared to the parental varieties and other crosses. Similarly, plant regeneration of F1 hybrids and F2 plants were higher than in both parents. The highest green plant regeneration frequency $(69.7 \%)$ was observed in F2 plants of $\mathrm{Bg} 90-2 \times \mathrm{Hu}$ lo tao cross. Higher regeneration frequencies of green plants, compared to albino plants, were observed with japonica parent and F1/ F2 plants of Bg 90-2 $\times \mathrm{Hu}$ lo tao cross. Although high frequency of green plants could be obtained from some of the F1 hybrids and F2 plants, green/ albino ratio for most of the genotypes remained low due to the high level of albino plant regeneration.

\section{Transplanting and growth of anther derived plants}

Anther derived green plants obtained from parents, F1 hybrids and F2 plants were acclimatized with approximately a mean percentage survival rate of 83 (Figure 1e, 1f). Spikelet fertility varied considerably with the genotype (Table 3 ). Out of the 278 plants that survived, 110 plants were completely sterile, 51 plants had 1-5\% spikelet fertility and 117 plants had more than $5 \%$ spikelet fertility.

\section{DISCUSSION}

The poor response of indica types to anther culture has been clearly established. Even among different genotypes of a particular ecotype, indica or japonica, considerable variation in pollen callusing and green plant regeneration has been observed with the genotypic effect being greater among the indica types (Silva, 2010). Results of this study agree with the above observations. Indica varieties had significantly low callus induction response and plant regeneration than the japonica variety. indica variety $\mathrm{Bg}$ 90-2 performed better than the other two indica varieties. This improved variety had indica type parentage. However, one or more parents of this variety might be of better anther culture responding indica type than the other parents.

Crossing of indica varieties with a japonica variety

Table 3: Transplantation survival and spikelet fertility of the anther derived plants from indica, japonica rice varieties, and their F1 hybrids and F2 plants

\begin{tabular}{|c|c|c|c|c|c|c|c|c|c|}
\hline \multirow{3}{*}{ Genotype } & \multicolumn{3}{|c|}{ Transplantation survival } & \multicolumn{6}{|c|}{ Plants with spikelet fertility } \\
\hline & \multirow{2}{*}{$\begin{array}{l}\text { Number of } \\
\text { plants } \\
\text { transferred }\end{array}$} & \multirow{2}{*}{$\begin{array}{c}\text { Number of } \\
\text { plants } \\
\text { survived }\end{array}$} & \multirow{2}{*}{$\begin{array}{c}\text { Percentage } \\
\text { survival }\end{array}$} & \multicolumn{2}{|c|}{$0 \%$} & \multicolumn{2}{|c|}{$1-5 \%$} & \multicolumn{2}{|c|}{$>5 \%$} \\
\hline & & & & No. & $\%$ & No. & $\%$ & No. & $\%$ \\
\hline \multicolumn{10}{|l|}{ Parents } \\
\hline Bg 90-2 & 11 & 9 & 81.8 & 1 & 11.1 & 2 & 22.2 & 6 & 66.6 \\
\hline Bg 379-2 & 0 & 0 & 0 & 0 & 0 & 0 & 0 & 0 & 0 \\
\hline Bg 94-1 & 0 & 0 & 0 & 0 & 0 & 0 & 0 & 0 & 0 \\
\hline $\mathrm{Hu}$ lo tao & 25 & 21 & 84.0 & 3 & 14.2 & 5 & 23.8 & 13 & 61.9 \\
\hline \multicolumn{10}{|l|}{ F1 hybrids } \\
\hline Bg 90-2 $\times$ Hu lo tao & 81 & 73 & 90.1 & 32 & 43.8 & 12 & 16.4 & 29 & 39.7 \\
\hline Bg $379-2 \times$ Hu lo tao & 61 & 54 & 88.5 & 26 & 48.1 & 9 & 16.6 & 19 & 35.1 \\
\hline Bg 94-1 × Hu lo tao & 47 & 32 & 68.0 & 18 & 56.2 & 6 & 18.7 & 8 & 25.0 \\
\hline \multicolumn{10}{|l|}{ F2 plants } \\
\hline Bg 90-2 $\times$ Hu lo tao & 41 & 35 & 85.3 & 12 & 34.2 & 5 & 14.2 & 18 & 51.4 \\
\hline Bg $379-2 \times$ Hu lo tao & 32 & 28 & 87.5 & 10 & 35.7 & 7 & 25.0 & 11 & 39.2 \\
\hline Bg 94-1 × Hu lo tao & 30 & 26 & 86.6 & 8 & 30.7 & 5 & 19.2 & 13 & 50.0 \\
\hline Total & 328 & 278 & & 110 & & 51 & & 117 & \\
\hline Mean percentage & & & 83.9 & & 39.5 & & 18.3 & & 42.0 \\
\hline
\end{tabular}


improves the callus induction frequency in anther culture. Significant positive heterosis was observed in callus induction in rice. It has been suggested that in rice, callus induction was mainly controlled by additive genetic effects (Yan et al., 1996). That the quality and frequency of callus induction and subsequent plant regeneration could be improved by selecting better responsive rice genotypes has been suggested (Niroula \& Bimb, 2009). Callus induction in rice is a quantitative trait controlled by multiple genes with additive effect. The callus induction process is under gametophytic control and the positive alleles would be strongly selected during the anther culture of F1 hybrids (Ping et al., 1997).

The plant regeneration frequency of F1 hybrids and F2 plants were better than both parents. In a similar study of androgenesis in indica $\times$ Basmati rice hybrids, high plant regeneration frequencies were observed from microspore derived calli of some of F1 hybrids and F2 plants as compared to their actual parents, supporting the above observations (Bishnoi et al., 2000). Although green plant regeneration from anther culture of indica cultivars was much lower than from japonica cultivars a considerable frequency of green plants from different crosses of indica cultivars have been obtained (Thuan et al., 2001).

The highest green plant regeneration frequency was observed in F2 plants of Bg 90-2 × Hu lo tao cross. This could be due to the fact that the segregating progeny has a range of favourable gene combinations that govern anther culture responses. According to Chu and Croughan (1989), from a practical stand point for rice anther culture, higher regeneration rates can be obtained from crosses involving a high and low parent by utilizing the F2 rather than the F1 generation for anther culture. A higher green plant regeneration than albino plants was observed with japonica parent and F1/ F2 plants of Bg 90-2 $\times \mathrm{Hu}$ lo tao cross. Although high frequency of green plants could be obtained from some of the F1 hybrids and F2 plants, green/ albino ratio for most of the genotypes remained low due to the high level of albino plant regeneration. The genotype appears to be the most important factor in androgenic albinism (Caredda \& Clement, 1999). According to Immonen et al. (1999), the use of doubled haploids is a production method that can be applied to a large number of genotypes, and a large number of green regenerants that are of high quality, displaying genetic variability of the cross can be produced. Although inheritance of anther culture response seems complex, crosses between high and low response types of barley has primarily shown additive effects (Hou et al., 1994). This agrees with the above results obtained. There have been several previous studies on the genetic control of anther culture in rice. Genetic additive effect was reported to be significant for callus induction, green plant regeneration and culture efficiency (Yan et al., 1996). Similarly, it has been found that the potential to introduce high responding ability into low lines was moderate, suggesting that the frequency of anther culture response might be improved by suitable hybridization and selection strategies (He et al., 2006). A genetic approach to improving plant regeneration from anther-derived callus in indica rice through sexual hybridization and selection has been suggested. (Silva, 2010). The higher heritability estimates for callus induction and culture efficiency suggest that relatively rapid genetic gain can be reached by transferring this trait from high culture ability to low culture ability germplasm (Yan et al., 1996). According to Silva (2010), irrespective of the method used, it was vitally important that such incorporation of genes should be recoverable in commercially advantageous rice varieties. The performance of F2 populations confirmed F1 additive effects. The F2 generation demonstrated that combining genes from different populations can sometimes have unexpected positive effects (Fenster \& Galloway, 2000). In general, F2 hybrids respond better to anther culture than F1 hybrids in indica, Basmati rice breeding (Rohilla et al., 1997). Better than expected performance of recombinant hybrids may be attributed to the chance bringing together of groups of alleles in combination. According to Fenster and Galloway (2000), the presence of epistasis or gene interaction may cause the positive effects.

High spikelet sterility was observed in anther culture derived F1 hybrids and F2 plants. According to Roy and Mandal (2005), spikelet sterility occurs in androclones under in vitro culture conditions, due to physiological factors and changes in gene distribution.

\section{References}

1. Bishnoi U. S., Jain R. K., Gupta K. R., Chowdhury V. K. \& Chowdhury J. B. (2000). High frequency androgenesis in Indica $\times$ Basmati rice hybrids using liquid culture media. Plant Cell, Tissue and Organ Culture 61(2): 153-159.

2. Bregitzer P. \& Campbell D. (2001). Genetic markers associated with green and albino plant regeneration from embryogenic barley callus. Crop Science 41: 173-179.

3. Caredda S. \& Clement C. (1999). Androgenesis and albinism in Poaceae: influence of genotype and carbohydrates. In: Anther and Pollen (eds. C. Clement, E. Pacini \& J. C. Andran), pp. 211-228. Springer Verlag, Berlin, Germany.

4. Chen C. C., Tsay H. S. \& Huang C. R. (1991). Factors affecting androgenesis in rice (Oryza sativa L.). In: Biotechnology in Agriculture and Forestry 14: Rice (ed. Y. P. S Bajaj.), pp. 193-211. Springer Verlag, Berlin, Germany. 
5. Chu Q. R., Cinscombe S. D. \& Cao H. X. (2002). Rice anther culture expedites variety improvement for Lousiana. Proceedings of the Second Temperate Rice Conference, Sacramento, California, USA, 13-17 June 1999 (eds. J. E. Hill \& B. Hardly). International Rice Research Institute, Los Banos, Philippines. pp. 395-401.

6. Chu Q. R. \& Croughan T. P. (1989). Genetics of plant regeneration in anther culture of rice (Oryza sativa L.). Review of Advances in Plant Biotechnology. In: (eds. A. Mujeeb - Kazi \& L. A. Sitch), pp. 217-227. International Rice Research Institute, Los Banos, Philippines.

7. Chu C. C. (1978). The N6 medium and its applications to anther culture of cereal crops. Proceedings of the Symposium on Plant Tissue Culture, Peking, China, 25-30 May. Science Press, Beijing, China pp. 43-50.

8. Croughan T. P. (1998). Anther culture for double haploid production. In: Plant Cell Tissue and Organ Culture: Fundamental Methods (eds. O. L. Gamborg \& G. C. Phillips), pp. 143-154. Narosa Publishing, New Delhi, India.

9. Croughan T. P. \& Chu Q. R. (1991). Rice (Oryza sativa L.). Establishment of callus cultures and the regeneration of plants. In: Biotechnology in Agriculture and Forestry 14: Rice (ed. Y. P. S. Bajaj), pp. 19-37. Springer Verlag, Berlin, Germany.

10. Fenster C. B. \& Galloway L. F. (2000). Population differentiation in an annual legume: genetic architecture. Evolution 54 (4): 1157-1172.

11. He T., Yang Y., Tu S. B., Yu M. Q. \& Li X. F. (2006). Selection of interspecific hybrids for anther culture of Indica rice. Plant Cell, Tissue and Organ Culture 86(2): 271-277.

12. Herath H. M. I., Bandara D. C., Samarajeewa P. K. \& Wijesundara D. S. A. (2009). Effect of low temperature pre-treatment on anther culture in selected indica, japonica rice varieties and their inter sub specific hybrids. Ceylon Journal of Science (Biological Sciences) 38(1): 11-26.

13. Herath H. M. I., Bandara D. C. \& Samarajeewa P. K. (2007). Effect of culture media for anther culture of indica rice varieties and hybrids of indica and japonica. Tropical Agricultural Research and Extension 10: 17-22.

14. Hou L., Ullrich S. E. \& Kleinhofs A. (1994). Inheritance of anther culture traits in barley. Crop Science 34: $1243-1247$.

15. Immonen S., Tauriainen A. \& Manninen O. (1999). Assessment of green regenerants from rye and triticale anther cultures. In: Anther and Pollen (eds. C. Clement, E. Pacini \& J. C. Andran), pp. 237-245. Springer Verlag, Berlin, Germany.
16. Mendis M. H., Hettiarachchi A., Dhanapala M. P. \& Ganashan P. (1993). Application of innovative techniques for rice breeding. In: Biotechnology in Agricultural Development (eds. P. Ganashan \& S. Balendira), pp. 1723. Department of Agriculture, Peradeniya.

17. Murashige T. P. \& Skoog F. (1962). A revised medium for rapid growth and bioassays with tobacco tissue culture. Physiologia Plantarum 15 (3): 473-497.

18. Narasimman R. \& Rangasamy S. R. (1993). Comparison of fertility between the F1, F2 and anther derived lines in the crosses of indica/ japonica and japonica/ indica in rice (Oryza sativa L.). Euphytica 66(1-2): 19-25.

19. Niroula R. K. \& Bimb H. P. (2009). Effect of genotype and callus induction medium on green plant regeneration from anther of Nepalese rise cultivars. Asian Journal of Plant Sciences 8(5): 368-374.

20. Ping H., Ying C., Lishuang S., Chaofu L. \& Lihuang Z. (1997). Inheritance of callus induction ability in rice anther culture. Chinese Science Bulletin 42(14): 1209-1211.

21. Reed S. M. (2002). Haploid cultures. In: Plant Tissue Culture Concepts and Laboratory Exercises (eds. R. N. Trigiano \& D. J. Gray), $2^{\text {nd }}$ edition, pp.285-290. CRC Press, New York, USA.

22. Rohilla J. S., Chowdhury J. B., Yadev N. R., Chowdhury V. K. \& Jain K. K. (1997). Anther culture of indica / basmati rice heterotic $\mathrm{F} 1$ and $\mathrm{F} 2$ hybrids and selection of desirable double haploid lines. International Rice Research Notes 22: $14-15$.

23. Roy B. \& Mandal A. B. (2005). Anther culture response in indica rice and variations in major agronomic characters among the androclones of a scented cultivar Karnal local. African Journal of Biotechnology 4 (3): 235-240.

24. Senadheera D., Zapatha-Aris F.J., Gregorio G. B., Alejar M. S., Cruz H.C., Padolina T. F. \& Galvez A. M. (2002). Development of the first salt-tolerant rice cultivar through indical indica anther culture. Field Crops Research 76: 103-110.

25. Silva T. D. (2010). Indica rice anther culture: can the impass be surpassed? Plant Cell, Tissue and Organ Culture 100(1):1-11.

26. Sopory S. K. \& Munshi M. (1996). Anther culture. In: In vitro Haploid Production in Higher Plants (eds. S. M. Jain, S. K. Sopory \& R. E. Veilleus), pp. 145-176. Kluwer Academic Publishers, The Netherlands.

27. Thuan O. T., Tuan V. D. \& Bongs B. B. (2001). Study on anther culture of $\mathrm{F} 1$ plants from crosses between aromatic and improved rice cultivars. Omonrice 9: 41-45.

28. Yan J., Xue Q. \& Zhu J. (1996). Genetic studies of anther culture ability in rice (Oryza sativa). Plant Cell, Tissue and Organ Culture 45(3): 253-258. 